\title{
LAPAROSCOPIC ASSISTED RADICAL CYSTOPROSTATECTOMY WITH Y-SHAPED ORTHOTOPIC ILEAL NEOBLADDER CONSTRUCTED WITH NON-ABSORBABLE TITANIUM STAPLES THROUGH A 5 CM PFANNENSTEIL INCISION
}

\author{
SIDNEY C. ABREU, FREDERICO I. MESSIAS, RENATO S. ARGOLLO, GLAUCO A. \\ GUEDES, MARDHEN B. ARAUJO, GILVAN N. FONSECA \\ Urological Hospital of Brasília (SCA, FIM, RSA, GAG) and Sections of Urology, Federal University of \\ Ceara (MBA) and Federal University of Goias (GNF), Brazil
}

\begin{abstract}
Introduction: We performed a laparoscopic radical cystoprostatectomy followed by constructing a Y-shaped reservoir extra-corporeally with titanium staples through a 5-cm muscle-splitting Pfannenstiel incision.

Surgical technique: Upon completion of the extirpative part of the operation, the surgical specimen was entrapped and removed intact through a 5-cm Pfannenstiel incision. Through the extraction incision, the distal ileum was identified and a $40 \mathrm{~cm}$ segment isolated. With the aid of the laparoscope, the ureters were brought outside the abdominal cavity and freshened and spatulated for approximately $1.5-\mathrm{cm}$. Bilateral double $\mathrm{J}$ ureteral stents were then inserted up to the renal pelvis and the ureters were directly anastomosed to the open ends of the limbs of the neobladder. Following this, the isolated intestinal segment was arranged in a Y shape with two central segments of $14 \mathrm{~cm}$ and two limbs of $6 \mathrm{~cm}$. The two central segments were brought together and detubularized, with two sequential firings of $80 \times 3.5 \mathrm{~mm}$ and $60 \times 3.5 \mathrm{~mm}$ non-absorbable mechanical stapler (Multifire GIA - US Surgical) inserted through an opening made at the lowest point of the neobladder on its anti-mesenteric border. The neobladder was reinserted inside the abdominal cavity and anastomosed to the urethra with intracorporeal laparoscopic free-hand suturing.

Conclusion: Although this procedure is feasible and the preliminary results encouraging, continued surveillance is necessary to determine the lithiasis-inducing potential of these titanium staples within the urinary tract.
\end{abstract}

Key words: bladder neoplasms; laparoscopy; cystectomy; urinary reservoirs; neobladder, surgical stapling

Int Braz J Urol. 2005; 31: 362-9

\section{INTRODUCTION}

Initially restricted to a few centers of excellence, the worldwide experience with laparoscopic radical cystectomy is clearly on the increase (1). Nowadays, the extirpative portion of the procedure does not represent a limiting factor and pure laparoscopic techniques are employed to expeditiously perform radical cystectomy and pelvic lymphadenectomy $(2,3)$. Nonetheless, there is a trend to perform the reconstructive procedures necessary to create the urinary diversion extracorporeally, using 
conventional open surgical technique in an attempt to reduce the overall surgical time (1). Recently, the Italian group from Piedmont has described an "easy, fast and reliable" technique to create an orthotopic Y-shaped ileal neobladder open surgically using nonabsorbable titanium staples (4). Based on these promising results, we sought to perform a laparoscopic radical cystoprostatectomy followed by the construction of a Y-shaped reservoir extracorporeally through a $5 \mathrm{~cm}$ muscle-splitting Pfannenstiel incision. Therefore maximizing the benefits of a minimally invasive approach including, decreased postoperative pain, shorter hospital stay, quicker recovery, better cosmesis without a significant increase in the operative time.

Herein, we describe the surgical technique and present our preliminary results with laparoscopic assisted radical cystoprostatectomy with Y-shaped orthotopic ileal neobladder using non-absorbable titanium staples.

\section{SURGICAL TECHNIQUE}

Laparoscopic radical cystoprostatectomy was performed using a 5-port transperitoneal approach, which has been described in detail elsewhere (1-3). Upon completion of this extirpative part of the operation, the surgical specimen was entrapped in a Lapsac and removed intact through a 5-cm muscle-splitting Pfannenstiel incision (Figure-1). Through the extraction incision, the distal ileum was identified and brought outside the abdominal cavity. Using conventional open

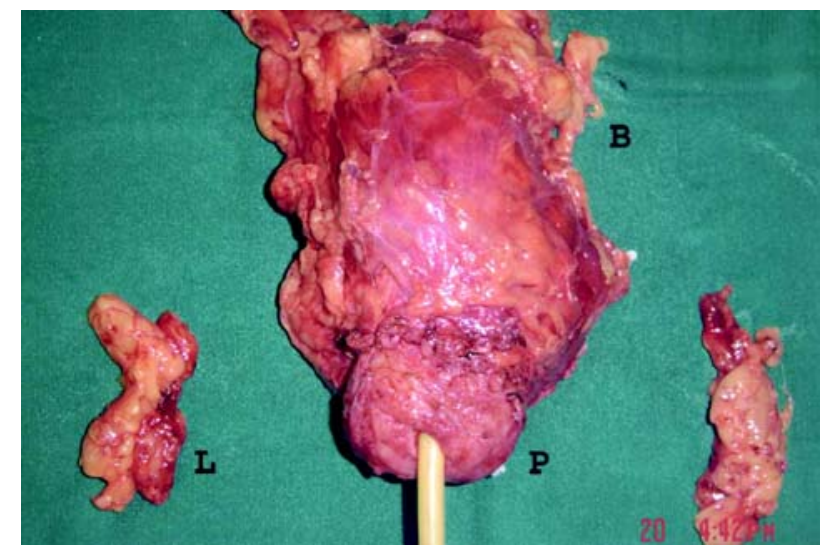

Figure 1 - Photograph showing intact surgical specimen: bladder $(B)$, prostate $(P)$ and lymph nodes $(L)$. surgical techniques, a $40 \mathrm{~cm}$ ileal segment was isolated 15 to $20 \mathrm{~cm}$ proximal to the ileocecal valve and a side-to-side entero-entero anastomosis was performed to re-establish bowel continuity. Through the laparoscope, the ureters, which were previously anchored to the anterior abdominal wall with a stay stitch, were identified and also brought outside the abdominal cavity. Laparoscopic viewing was used to ensure the ureters were not twisted or rotated. Subsequently, the ureters were freshened and spatulated for approximately $1.5-\mathrm{cm}$. Bilateral, $6 \mathrm{~F}$ double $\mathrm{J}$ ureteral stents were then inserted up to the renal pelvis and the ureters were directly anastomosed to the open ends of the limbs of the neobladder using running sutures of 4-0 PDS (Figure-2). Following this, the isolated intestinal segment was arranged in a Y shape with 2 central segments of $14 \mathrm{~cm}$ and two limbs of $6 \mathrm{~cm}$. The 2 central segments
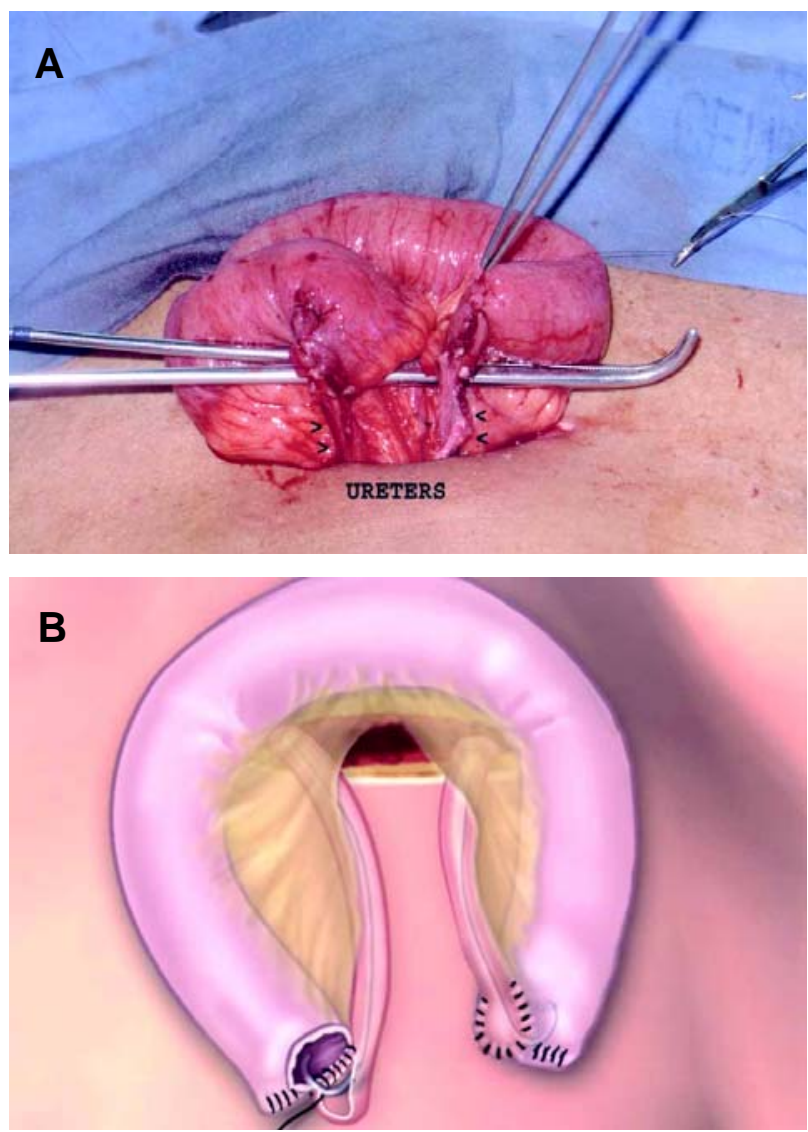

Figure 2 - A) Photograph showing the ureters (arrowheads) being directly anastomosed to the open ends of the limbs of the neobladder; B) Schematic drawing. 
were brought together and detubularized with 2 sequential firings of a $80 \times 3.5 \mathrm{~mm}$ and $60 \times 3.5 \mathrm{~mm}$ nonabsorbable mechanical stapler (Multifire GIA - US Surgical) inserted through an opening made at the lowest point of the neobladder on its anti-mesenteric border (Figure-3). The recently created neobladder was reinserted inside the abdominal cavity and the $5-\mathrm{cm}$ Pfannenstiel incision was closed. Pneumoperitoneum was re-established and the Y-neobladder was anastomosed to the urethra with four interrupted 2-0 monocryl sutures over a 22F Foley catheter, using intracorporeal laparoscopic free-hand suturing. The 2 limbs of the neobladder were then fixed to the psoas muscles. A suction drain was left in the deep pelvis.

\section{PATIENTS AND RESULTS}

Two male patients diagnosed with muscle invasive transitional cell carcinoma of the bladder consented and were scheduled to a laparoscopic assisted radical cystoprostatectomy with orthotopic Yshaped ileal neobladder. Metastatic workouts with chest $\mathrm{x}$-rays and abdominal CTs were negative in both patients. There were no signs of enlarged pelvic nodes. Demographic, intraoperative and postoperative data are presented in Table-1.

A retrograde cystogram was performed with $200 \mathrm{cc}$ of contrast on postoperative day 3 and postoperative day 7 in the first and second cases respectively, showing no signs of contrast media extravasation (Figure-4). In both cases, bladder catheter was removed on postoperative day 21 . In both cases, cystoscopic viewing in the second postoperative month did not reveal any stones at the staple lines, which were almost entirely covered by the intestinal mucosa, except by a single spot where 3 staples could be visualized in the first case (Figure-5). We used a smaller 5-cm Pfannenstiel incision (Figure-6) to perform the bowel work, the Y-shaped orthotopic ileal neobladder and the ureteral re-implantation, thus further improving cosmesis and perhaps further decreasing surgical morbidity. Pathology revealed a T2G3 transitional cell carcinoma (TCC) of the bladder with negative surgical margins in the first case and a T3aG3 TCC of the bladder in the second case. Lymph nodes were negative in both cases.
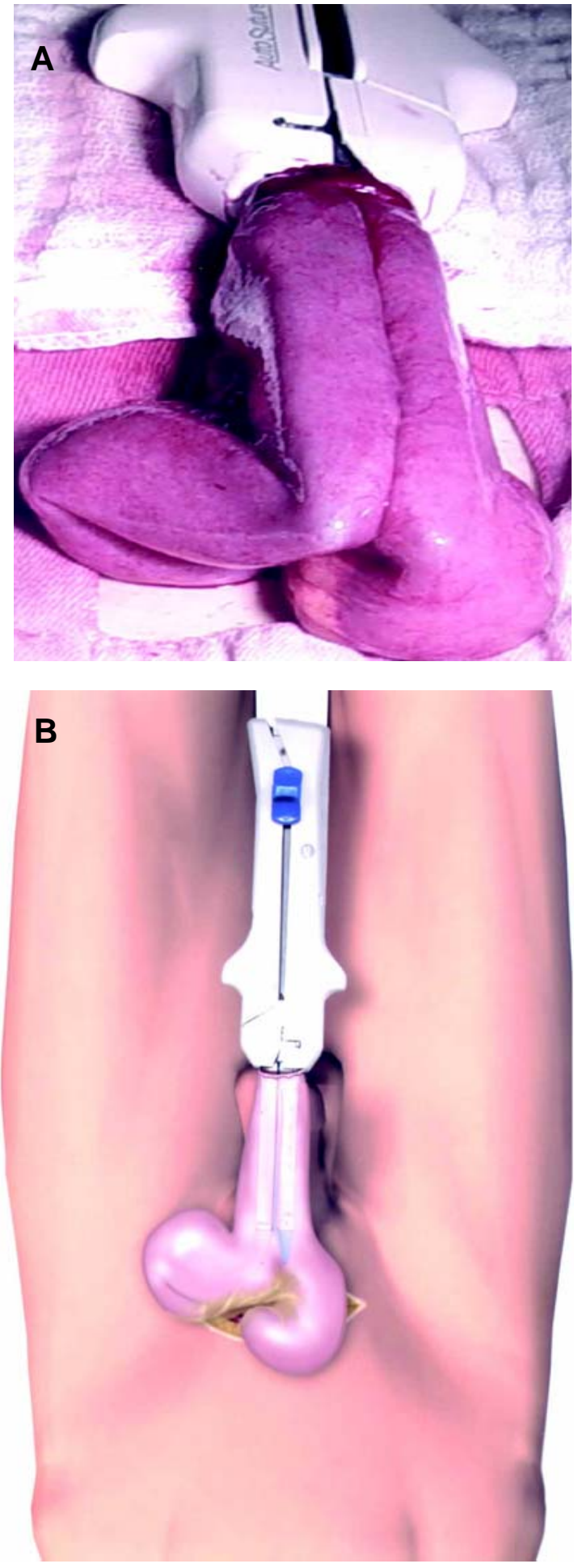

Figure 3 - A) Photograph showing the Y-shaped neobladder being created with non-absorbable titanium staples; B) Schematic drawing. 
Table 1 - Demographics, intraoperative and postoperative data.

\begin{tabular}{lcc}
\hline & Case \#1 & Case \#2 \\
\hline Age (years) & 64 & 59 \\
ASA & 2 & 2 \\
OR Time & 5.0 & 6.5 \\
$\quad$ Total (hrs) & 3.5 & 4.5 \\
- Radical cystectomy and pelvic lymphadenectomy (hrs) & 45 & 40 \\
- Bowel work and neobladder construction (min) & 25 & 25 \\
Urethro-intestinal anastomosis (min) & 20 & 55 \\
Blood loss (cc) & 250 & 1 \\
Blood transfusion (units) & - & 3 \\
Complications & 2 & 7 \\
Oral fluids intake (POD) & 3 & 7 \\
Cystogram without leak (POD) * & 5 & \\
Hospital stay (days) & & \\
\hline
\end{tabular}

* 200 cc of contrast

\section{COMMENTS}

Over the past decade, a few studies have reported that the use of non-absorbable titanium staples inside the urinary tract may be safe and secure (4-9). The corrosive resistant nature, low toxicity and excellent tissue and fluid biocompatibility of titanium may explain why such non-absorbable staples are well tolerated here. In fact, in 1993, Kerbl and colleagues from Washington University have reported the initial encouraging laboratory and clinical data with laparoscopic stapled (titanium staples) bladder closure (4). However, this article was criticized by reviewers at that time, who used comments such as: “...just because it is new, rapid and easy to use does not mean that it should be used in a cavalier fashion;" and "... sutures are less expensive than staples and less likely to result in stone formation." However, in 2000 this same group discussed its follow up research conducted over a more than 10 -year period, and attesting to the safety of using non-absorbable titanium staples to secure the bladder cuff during laparoscopic nephroureterectomy (5), and demonstrating that no stones have ever been observed at the titanium staple line even when the staples were not entirely covered by the vesical mucosa.
In 2004, an Italian group from Piedmont reported an acceptable $6 \%$ rate of stones formation when fifty Y-shaped orthotopic ileal neobladders were created in open surgical procedures with non-absorbable titanium staples in a mean follow up of 20 months (total follow up range: from 8 to 47 months) (7). Encouraged by the Fontana and colleagues study, we recently performed and documented the first orthotopic ileal neobladder constructed totally intracorporeally using non-absorbable titanium staples exclusively (8). Although the procedure was possible, it took a long time (approximately 10 hours) and it required a considerable amount of disposables (8 stapler loads) to complete it. In an attempt to overcome these difficulties, we sought to incorporate Fontana's idea of creating an ileal neobladder with non-absorbable titanium staples with the technique discussed by Basillote and colleagues of laparoscopic assisted radical cystectomy (2). Basillote et al. have demonstrated that when radical cystectomy is performed laparoscopically and the neobladder is performed open surgically with conventional techniques through a Pfannenstiel incision, the patient has the benefits of a minimally invasive approach, including decreased post-operative pain and quicker recovery, without a significant increase in operative time (2). Therefore, 


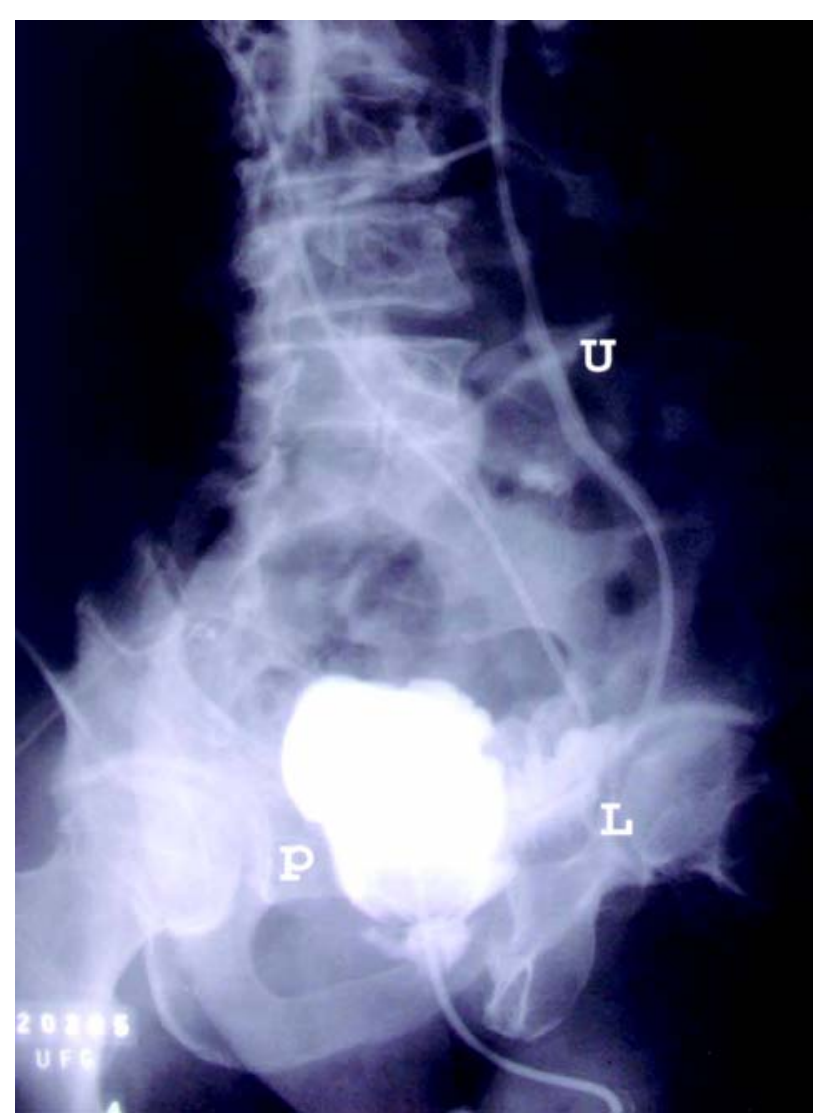

Figure 4 - Retrograde pouchgram with 200 cc performed on post-operative day 7 showing no signs of contrast extravasation, but free reflux of contrast from the limbs $(L)$ of the pouch $(P)$ to the ureters $(U)$.

we performed 2 cases of laparoscopic assisted radical cystoprostatectomy wherein the Y-shaped orthotopic ileal neobladder was created extracorporeally using non-absorbable titanium staples.

Although longer follow-up is required to fully evaluate the safety of this approach, we believe that our initial results are encouraging. It is also worth noting the mean overall surgical time of 5.7 hours, mean blood loss of $375 \mathrm{cc}$ and mean total hospital stay of 5 days. Moreover, over a very short period ranging from 3-7 days post-operatively, we were able to demonstrate that there were no signs of contrast extravasation during retrograde pouchgram performed with $200 \mathrm{cc}$ of contrast. Furthermore, cystoscopy performed in the second post-operative month did not reveal any stones at the staple line, and only a few
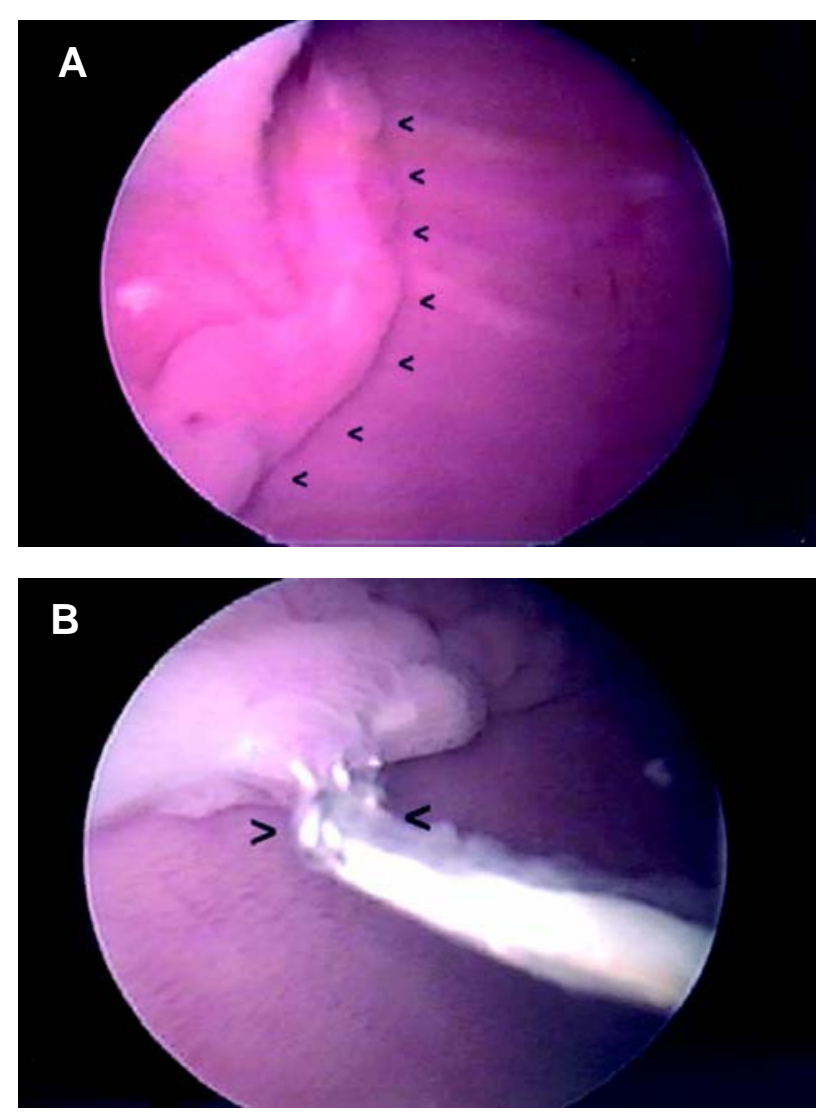

Figure 5 - A) Photograph showing the staple line (multiple arrows) covered by the intestinal mucosa; and B) Single spot where 3 staples were viewed within the reservoir (there is mucus adherent to the staples).

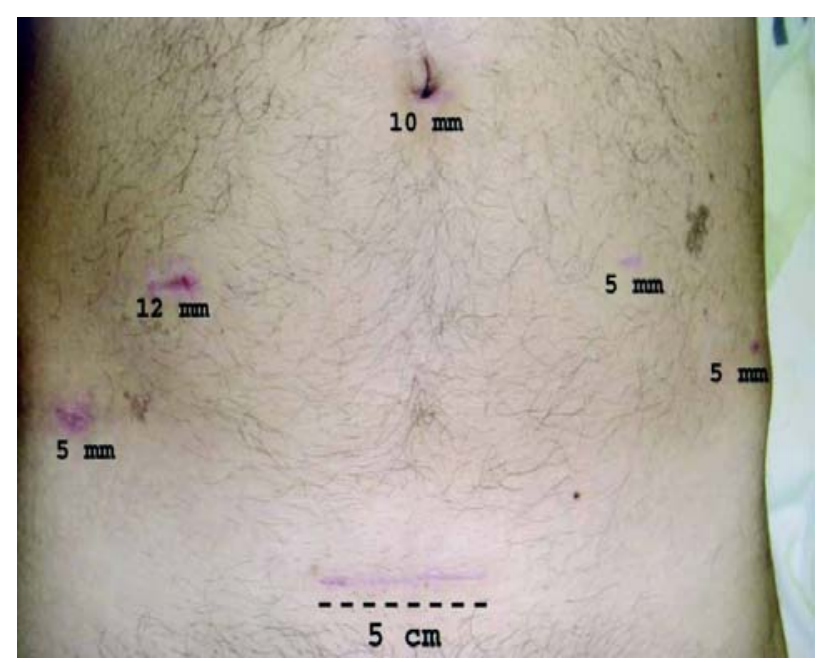

Figure 6 - Photograph of port site incisions and 5-cm musclesplitting Pfannenstiel incision. 
staples were visualized within the neobladder while the remainder of the staple line was recovered by the intestinal mucosa.

We differed from the technique described by Basillote et al., who used a 15-cm Pfannenstiel incision to perform bowel work, Studer orthotopic ileal neobladder, ureteral re-implantation and the urethralileal anastomosis (2). As reported, we used a smaller 5-cm Pfannenstiel incision to perform the bowel work, Y-shaped orthotopic ileal neobladder and ureteral reimplantation, thus further improving cosmesis and perhaps further decreasing surgical morbidity. In our approach, the urethral-ileal anastomosis is performed using free-hand laparoscopic suturing after the recently fashioned neobladder is pushed back into the abdominal cavity, the extraction incision is closed and pneumoperitoneum is re-established.

Moreover, our approach to the bilateral ureteral re-implantation was different from that originally described by Fontana et al. In their report, the ureters were spatulated anteriorly and were anastomosed to the dorsal aspect of the two limbs of the $\mathrm{Y}$ with 5-0 sutures using the direct (end-to-side) Nesbit technique. Subsequently, the previously placed ureteral stents were brought out through the distal portions of each chimney and then through the anterior abdominal wall. In our procedure, the ureters were freshened and spatulated for approximately $1.5-\mathrm{cm}$, and then directly anastomosed (end-to-end) to the already open ends of the 2 limbs with 4-0 sutures over previously placed double J ureteral stents, resulting in a wide patent anastomosis. We thus avoided the presence of external ureteral stents and did not have to close the open ends of the limbs or create 2 new openings in the chimneys in order to implant the ureters, thus saving some time and perhaps decreasing the chances of developing a urinary fistula in the reservoir.

\section{CONCLUSION}

Laparoscopic assisted radical cystoprostatectomy with Y-shaped orthotopic ileal neobladder constructed using non-absorbable titanium staples through a 5-cm Pfannenstiel incision is feasible and our preliminary results are encouraging. Continued surveillance will be necessary to determine the lithiasis-inducing potential of these titanium staples within the urinary tract.

\section{REFERENCES}

1. Moinzadeh A, Gill IS: Laparoscopic radical cystectomy with urinary diversion. Curr Opin Urol. 2004; 14: 83-7.

2. Basillote JB, Abdelshehid C, Ahlering TE, Shanberg AM: Laparoscopic assisted radical cystectomy with ileal neobladder: a comparison with the open approach. J Urol. 2004; 172: 489-93.

3. Abreu SC, Gill I: Laparoscopic radical cystectomy. In: Cadeddu J, (ed.), Laparoscopic Urologic Oncology. Totowa, New Jersey. Humana Press Inc. 2003; p. 297-304.

4. Kerbl K, Chandhoke P, McDougall E, Figenshau RS, Stone AM, Clayman RV: Laparoscopic stapled bladder closure: laboratory and clinical experience. J Urol. 1993; 149: 1437-9; discussion 1439-40.

5. Shalhav AL, Dunn MD, Portis AJ, Elbahnasy AM, McDougall EM, Clayman RV: Laparoscopic nephroureterectomy for upper tract transitional cell cancer: the Washington University experience. J Urol. 2000; 163: 1100-4.

6. Grubb RL 3rd, Sundaram CP, Yan Y, Chen C, McDougall EM, Clayman RV: Use of titanium staples during upper tract laparoscopic reconstructive surgery: initial experience. J Urol. 2002; 168: 1366-9.

7. Fontana D, Bellina M, Fasolis G, Frea B, Scarpa RM, Mari M, et al.: Y-neobladder: an easy, fast, and reliable procedure. Urology. 2004; 63: 699-703.

8. Abreu, SC, Fonseca G, Gadelha JB: Laparoscopic radical cystectomy with intracorporeally constructed $\mathrm{Y}$ shaped ileal neobladder using non-absorbable titanium staples exclusively. Urology. (in press), 2005.

9. Abreu SC, Fonseca G, Gadelha JB: Re: Y-neobladder an Easy, Fast and Reliable Procedure. J Urol (in press), 2005.

Received: March 3, 2005

Accepted after revision: June 10, 2005 


\section{EDITORIAL COMMENT}

The authors are to be congratulated for achieving the advanced laparoscopic procedure of radical cystoprostatectomy. This procedure has started to gain interest worldwide, with more institutions performing the procedure. As with any new oncologic procedure, one must look at the outcomes in order to assess efficacy of the procedure. The margin status must be reported together with surveillance followup imaging studies. In the open cystectomy literature, 5 year survival is $87-89 \%$ for organ confined node negative disease compared to $50-62 \%$ for locally extravesical non-metastatic disease (1).

The limits of an extended pelvic lymph node dissection have been another area of discussion. An extended lymph node dissection should include the distal para-aortic and paracaval lymph nodes as well as the pre-sacral nodes, known anatomic sites of lymph node drainage from the bladder. An extended dissection may provide a survival advantage in patients with node-positive and node-negative tumors without significantly increasing the morbidity or mortality of the surgery (2). Recently, a multi-institutional study reviewed their series of where positive lymph nodes were most commonly found (3). This mapping study demonstrated positive lymph nodes were found most commonly in the obturator spaces and adjacent to the iliac vessels. Interestingly, $16 \%$ of lymph node metastases also included nodes above the aortic bifurcation, whereas $8 \%$ of lymph node metastases involved the presacral region.

Performing the ureteral reimplantation into the neobladder extracorporeally is a reasonable method to decrease operative time; however, one must be conscious of avoiding excessive tension when the ureters are pulled up through the incision, as this can lead to early strictures.

The use of non-absorbable titanium staples to create the neobladder is a balance between time saving maneuvers and decreasing the morbidity of the procedure. While stones have been reported at a rate of only $6 \%$ where titanium staples were used to construct the neobladder, one wonders if this is comparable with the baseline stone formation rate where mucus is a nidus of stone formation.

Clearly, laparoscopic radical cystectomy will continue to play a role in urologic oncology. It is currently at the infancy of institutions' experience, and differs from the experience of laparoscopic radical prostatectomy in that fewer cases are performed due to the incidence of muscle invasive transitional cell carcinoma compared to prostate cancer, as well as the average age and health status of the patients. Longer follow-up outcome data however is needed in order to insure optimal results.

\section{REFERENCES}

1. Stein JP, Lieskovsky G, Cote R, Groshen S, Feng AC, Boyd $S$ et al.: Radical cystectomy in the treatment of invasive bladder cancer: long term results in 1,054 patients. J Clin Oncol. 2001; 19: 666-75.

2. Stein JP, Skinner DG: The role of lymphadenectomy in high-grade invasive bladder cancer. Urol Clin North Am. 2005; 32: 187-97.

3. Leissner J, Ghoneim MA, Abol-Enein H, Thuroff JW, Franzaring L, Fisch M, et al.: Extended radical lymphadenectomy in patients with urothelial bladder cancer: results of a prospective multicenter study. $\mathbf{J}$ Urol. 2004; 171: 139-44.

Dr. Benjamin R. Lee

Laparoscopy Section, Department of Urology Long Island Jewish Medical Center New Hyde Park, New York, USA 


\section{EDITORIAL COMMENT}

The authors should be commended for presenting a hybrid procedure combining a novel open technique, the Y-shaped orthotopic ileal neobladder, and a minimally invasive laparoscopic approach. As with any hybrid procedure, the urologist will need broad surgical skills. Urologists using this approach must possess advanced laparoscopic skills, as they must perform a laparoscopic cystoprostatectomy and complete the urethrovesical anastomosis laparoscopically. Similarly, the creation of the Yshaped neobladder requires experience with urinary diversion, and specifically care must be taken to avoid twisting or rotating the ureter or blood supply to the neobladder during the procedure through the $5 \mathrm{~cm}$ incision.

It is evident that careful patient selection will be critical when using this technique. Patients having prior abdominal procedures, or with aggressive disease may be better suited to an alternative approach. Similarly, morbidly obese patients may prove challenging, specifically when managing the open neobladder creation through the smaller Pfannenstiel incision. Although convalescence data and long-term follow-up are lacking, this procedure should prove effective and beneficial. The authors diligently state the need for long-term follow-up of the neobladder for stone disease, although most experts would agree this would not be problematic.

In 2005, cystoprostatectomy and neobladder creation remains an open operation in most centers, despite the fact that many bladder cancer patients would benefit from the improved convalescence and cosmesis of the minimally invasive approach. Moreover, a more rapid recovery would be beneficial for bladder cancer patients requiring adjuvant chemotherapy. This report adds nicely to the growing body of literature supporting minimally invasive cystoprostatectomy and urinary diversion.

Dr. Stephen Y. Nakada Professor and Chairman of Urology University of Wisconsin Madison, Wisconsin, USA 